A. Pramesh Rao, G. Swarup and Gopal-Krishna, eds.

\title{
GMRT Observations of the Galactic Centre Region
}

\author{
Subhashis Roy and A. Pramesh Rao \\ National Center for Radio Astrophysics, TIFR, Pune, India
}

\section{Introduction}

We have started a project to study the Galactic Centre region using the GMRT in all it's frequency bands. For the first time, Galactic Centre have been imaged with high resolution at $610 \mathrm{MHz}$. Also, at $325 \mathrm{MHz}$, we have observed a few of the suspected supernovae remnants (SNRs) close to the Galactic Centre. Here we present some of the preliminary results of our 610 and $325 \mathrm{MHz}$ observations.

\section{1. $610 \mathrm{MHz}$ observation of the Galactic Centre:}

Previous high resolution studies of the central region have been carried out at 20 $\mathrm{cm}$ (Yusef-Zadeh 1984) and also at $90 \mathrm{~cm}$ (Pedlar et al. 1989, Anantharamaiah et al. 1991). However, this region has not been observed before at $49 \mathrm{~cm}$.

Here we present only the central $7^{\prime}$ region which contains the H-II region Sgr-A West, the SNR Sgr-A East and the extended halo of around $7^{\prime}$ around it (Fig.1). We found Sgr-A* to be visible in the $49 \mathrm{~cm}$ map, which disappears at $90 \mathrm{~cm}$ (Pedlar et al.1989) due to free-free absorption by the Sgr-A West HII region. We estimate a total flux of $1.1 \pm 0.2 \mathrm{Jy}$ from it. The detection indicates, if the Sgr-A* is located within the Sgr-A West complex, then the Sgr-A West region becomes optically thick between 49 and $90 \mathrm{~cm}$.

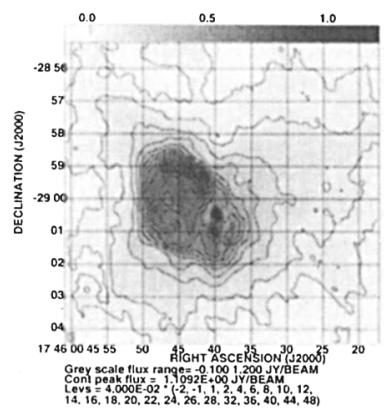

Figure 1. at $91 \mathrm{~cm}$

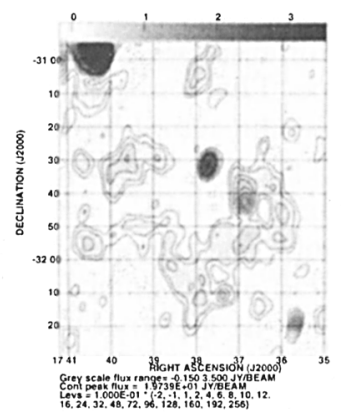

(b) G356.8-0.1, new SNR

(a) Galactic Centre at $49 \mathrm{~cm}$. \section{r}


G356.8-0.1: We observed a field centered on G 356.8-0.1 such that the suspected SNRs, G356.6+0.1, G356.3-00.3 and G357.1-00.2 falls within the field of view of the antennas. In our map (Fig. 2), all three suspected SNRs appear to be parts of a much bigger shell. Morphology strongly suggests G357.1-00.2 to be a part of the bigger shell at G356.8-00.1. Also, G357.1-00.2 shows polarised emission (Gray et al. 1996). This shows synchrotron radiation and indicates G356.8-00.1 to be an SNR. It has an angular size of $70^{\prime} \times 80^{\prime}$. We estimate a total flux of $30 \mathrm{Jy}$. However, this should be taken as an underestimate.

G3.1-00.6: Our low resolution map (Fig. 3.(a)) identifies the shell structure of this source. In high resolution (Fig. 3.(b)), diffuse emission from the western side of the shell is evident. This suggests a plerionic component of emission. High resolution map shows a filament at the eastern side of the SNR, which seems to be part of the shell at low resolution. Polarised emission upto $20 \%$ have been detected from it by us in the NVSS map (Condon et al. 1998). This filament has a spectral index of $-0.9 \pm 0.15$ between 843 and $325 \mathrm{MHz}$. Steep spectral index and polarised emission identifies G3.1-00.6 to be an SNR. We estimate a total flux of $18 \mathrm{Jy}$, which is the lower limit.
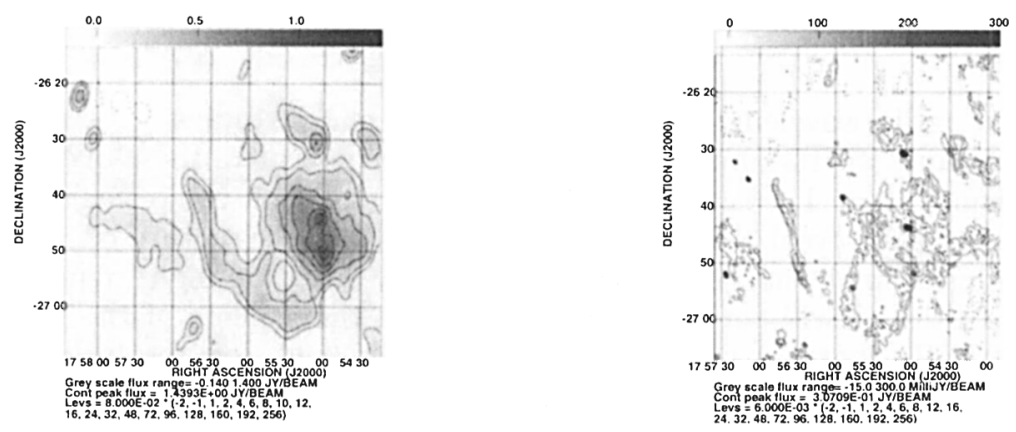

Figure 2. (a) G3.1-0.6 in low resolution (b) G3.1-0.6 in high resolution

\section{References}

Anantharamaiah, K.R., et al., 1991, MNRAS, 249, 262.

Condon, J. J. et al. 1998, AJ, 115, 1693.

Pedlar, A., et al., 1989, Ap.J., 342, 769.

Yusef-Zadeh, F., Morris, M. \& Chance, D., 1984, Nature, 310, 557.

Gray, A.D., 1994, MNRAS, 270, 847.

Gray, A.D., 1996, In:ASP Conf. Ser., Vol.102, p-443. 